\title{
ORIGINAL ARTICLE \\ Dehydration affects spinal cord cross-sectional area measurement on MRI in healthy subjects
}

\author{
C Wang ${ }^{1,2}$, RC Tam ${ }^{2,3}$, E Mackie ${ }^{2}, \mathrm{DKB} \mathrm{Li}^{2,3}$ and AL Traboulsee ${ }^{2}$
}

\begin{abstract}
Study design: This was a prospective cohort observational study. Objective: To determine the effect of dehydration and rehydration on spinal cord cross-sectional area (CSA) measurement on magnetic resonance imaging (MRI).

Setting: MRI Research Centre, University of British Columbia, Canada.

Methods: Ten healthy subjects (aged 21-32 years) were scanned on a 3T MRI scanner at four time points: (1) baseline, (2) rescan after $1 \mathrm{~h},(3)$ the next day after fasting for a minimum of $14 \mathrm{~h}$ and (4) after rehydration with $1.5 \mathrm{I}$ of water over the course of $1 \mathrm{~h}$. Two independent, established semi-automatic CSA measurement techniques (one based on two-dimensional (2D) edge detection, the other on three-dimensional (3D) surface fitting) were applied to a 3D T1-weighted scan of each subject at each time point, with the operator blinded to scan order. The percentage change in CSA from baseline to each subsequent time point was calculated. One-tailed paired $t$-tests were used to assess the significance of the changes from baseline.
\end{abstract}

Results: A decrease in CSA following dehydration was detected by both measurement methods, with a mean change of $-0.654 \%$ (s.d. $=0.778, P<0.05$ ) and $-0.650 \%$ (s.d. $=1.071, P<0.05)$ for the first and second methods, respectively.

Conclusion: Dehydration can confound CSA measurements on MRI. The magnitude of the effect is significant relative to short-term pathological changes that have been observed in diseases such as multiple sclerosis.

Spinal Cord (2014) 52, 616-620; doi:10.1038/sc.2014.66; published online 29 April 2014

\section{INTRODUCTION}

Magnetic resonance imaging (MRI) is commonly used to measure spinal cord atrophy in studies of neurodegenerative diseases such as multiple sclerosis (MS). ${ }^{1,2}$ Atrophy of the spinal cord, in particular the cervical cord, ${ }^{3}$ is believed to contribute to physical disability in MS. ${ }^{2,4}$ Significant correlations between the cross-sectional area (CSA) of the cord and physical disability in MS patients have been found in previous cross-sectional and longitudinal studies. ${ }^{5-7}$ However, there have been contradictory findings in patients with the relapsing remitting form of MS (RRMS), which typically precedes a more progressive form. A number of studies have found that cord CSA is not reduced in RRMS patients ${ }^{8,9}$ and could be used to distinguish progressive MS from RRMS patients, ${ }^{8}$ whereas others have shown that cord atrophy is detectable in RRMS patients. ${ }^{10}$

The inconsistent findings of cord involvement in RRMS may be because of many reasons. The average CSA of a normal adult cervical cord is $\sim 80 \mathrm{~mm}^{2}$. Most current MRI studies of the cord use a spatial resolution of $\sim 1 \mathrm{~mm}^{2}$ in each plane, which is $\sim 1 \%$ of the cord CSA. As atrophy is a slow process, with an annual atrophy rate of about $-1.6 \%$ observed in secondary progressive MS patients, ${ }^{11}$ a resolution of $1 \mathrm{~mm}^{2}$ can be considered coarse, and a high methodological sensitivity is essential to accurately estimate the true rate of change on current MRI data. A number of reproducible image analysis methods of measuring CSA are now in use; ${ }^{12-14}$ however, other factors may still confound CSA measurement.

The conventional assumption is that cord volume does not have transient variation, which needs to be reconsidered in light of evidence that brain volume has been shown to be affected by normal physiological variations and inflammatory processes. ${ }^{15,16}$ In particular, hydration status can affect brain morphology as observed on MRI in previous studies. ${ }^{16-20}$ Duning et al. ${ }^{16}$ reported that a cohort of 20 healthy volunteers showed a significant decrease in brain volume of $0.55 \%$ (s.d. $=0.69$ ) after dehydration by restricted fluid intake for $16 \mathrm{~h}$ and an increase of $0.72 \%$ (s.d. $=0.21$ ) after rapid rehydration. Kempton et al. ${ }^{18}$ observed a significant increase in ventricular volume following dehydration via a thermal exercise protocol in two studies of seven ${ }^{18}$ and ten ${ }^{19}$ healthy subjects. The change in hydration status can potentially also affect the size of the spinal cord which, similar to the brain, also has high water content.

Considering the small magnitude of cord atrophy in diseases such as MS, the question of whether cord CSA measurement is susceptible to dehydration requires examination. The goal of this study is to estimate how much variation in CSA can be expected due to dehydration to the degree that would not be considered unusual in daily functioning.

${ }^{1}$ Department of Electrical and Computer Engineering (Biomedical Engineering Program), University of British Columbia, Vancouver, British Columbia, Canada; ${ }^{2}$ Division of Neurology, Department of Medicine, University of British Columbia, Vancouver, British Columbia, Canada and ${ }^{3}$ Department of Radiology, University of British Columbia, Vancouver, British Columbia, Canada

Correspondence: C Wang, MS/MRI Research Group, Djavad Mowafaghian Centre for Brain Health, 2215 Wesbrook Mall, 3rd Floor, Room 3450-B, Vancouver, British Columbia, Canada V6T 2B5.

Email: wchunfang@gmail.com

Received 8 November 2013; revised 27 March 2014; accepted 1 April 2014; published online 29 April 2014 


\section{MATERIALS AND METHODS}

Subjects and MRI protocol

The subjects recruited for this study comprised 10 volunteers, aged 21-32 years, with no symptoms of neurological disorders or spine problems. The subjects gave informed consent in accordance with institutional regulations. Images were acquired using a Philips Achieva 3T MRI scanner (Philips Medical Systems, Best, The Netherlands) with a dedicated cervical spine receiver coil. The sequence is a sagittal 3D T1-weighted turbo field echo sequence with parameters $\mathrm{TR}=8.2 \mathrm{~ms}, \mathrm{TE}=3.8 \mathrm{~ms}$, flip angle $=8^{\circ}$, pixel spacing $=0.976$ $\mathrm{mm} \times 0.976 \mathrm{~mm}$, slice thickness $=1 \mathrm{~mm}$ and dimensions $=256 \times 256 \times 60$ pixels.

\section{Dehydration and rehydration protocol}

We employed a similar dehydration and rehydration protocol to that used by Duning et al. ${ }^{16}$ who studied the effect on whole-brain volume. For each subject, MR scans were obtained at four time points over 2 days: (1) baseline, (2) rescan after $1 \mathrm{~h}$ to measure reproducibility, (3) the next morning, after fasting (solid and liquid) for at least $14 \mathrm{~h}$ and (4) after drinking 1.51 of water over the course of $1 \mathrm{~h}$. The subjects were asked not to exercise strenuously during the 2 days of their participation in the study.

\section{MRI analysis}

The cord CSA in each scan was measured using two established semiautomatic methods. Two methods were used to cross-validate each other and to estimate the variability because of the choice of measurement method. One is our implementation of the technique by Tench et al..$^{13}$ The other is a method by Horsfield et al., ${ }^{14}$ which is available as part of the Jim software package (Version 6.0, Xinapse System, Kettering, UK; www.xinapse.com). The scans obtained at all four time points were anonymized and randomized, and image analysis was carried out in a blinded manner.

\section{Tench Method (2D edge detection)}

In the approach by Tench et al., ${ }^{13}$ the user interacts with the software by identifying an anatomical landmark on the spine in a sagittal view, and then segmenting a number of consecutive axial slices while being guided by an edge map that is produced by an edge detector on each 2D slice, as shown in Figure 1b). The operator places a seed point inside the cord on each slice and initiates a region-growing process, which is bounded by the contour as shown in Figure 1c). In the present study, we used a single sagittal landmark on the most inferior and posterior point of the $\mathrm{C} 2 / \mathrm{C} 3$ intervertebral disc, and the eight slices superior to the landmark were used to compute an average CSA.

For each segmented axial slice, all of the pixels strictly inside the cord have an area value equal to the pixel size $\left(1 \mathrm{~mm} \times 0.976 \mathrm{~mm}=0.976 \mathrm{~mm}^{2}\right)$, whereas each boundary pixel is assigned a value that is a fraction of the pixel size, modeled as partial volume between the cord and surrounding cerebrospinal fluid (CSF). The partial volume fraction $f$ is calculated using the equation $f=\left(i_{\text {edge }}-i_{\text {CSF }}\right) /\left(i_{\text {cord }}-i_{\text {CSF }}\right)$, where $i_{\text {edge }}$ is the intensity of the boundary pixel, and $i_{\mathrm{CSF}}$ and $i_{\text {cord }}$ are the intensities of the CSF and cord sampled on either side of the boundary pixel.

The CSA for each slice is calculated by summing the contributions from the interior and boundary pixels. A correction factor, calculated as the cosine of the angle between the medial axis of the cord and the axis perpendicular to the axial plane, is then applied to the CSA value to compensate for the fact that the cord is rarely perfectly perpendicular to the axial image plane.

\section{Horsfield method (3D surface fitting)}

In the method by Horsfield et al. ${ }^{14}$ the operator first performs angle correction by rotating the volume so that its edges are parallel to the cord in the region of interest ( $\mathrm{C} 1 \sim \mathrm{C} 2$ in the current study). Then the operator places landmarks at the center of the cord on the most superior and inferior axial slices of the $\mathrm{C} 1 \sim \mathrm{C} 2$ region, and on every 10th slice in-between. The software then uses these landmarks to automatically initialize a 3D surface and segment the spinal cord by fitting the surface to the image. There are three key parameters in the algorithm related to cord size and shape ('nominal cord diameter', 'number of shape coefficients' and 'order of longitudinal variation'), which can be used to influence the fitting process. We used the default values in our analysis because the study is of healthy subjects and the segmentations appeared to be visually accurate.

The total cervical cord volume was calculated by the software as an integral on the final fitted surface model. The average cervical cord CSA was then derived as the total volume divided by the length of the segmented region.

\section{Statistical analysis}

The percentage changes in CSA from baseline to the other three time points were calculated for each patient, and the mean change of the group was used to determine the effect of scan-rescan, dehydration and rehydration. The statistical significance of the group changes was assessed using paired onetailed $t$-tests, with $P<0.05$ as the threshold.

\section{Statement of ethics}

We certify that all applicable institutional and governmental regulations concerning the ethical use of human volunteers were followed during the course of this research.

\section{RESULTS}

Measured using the Tench method, the cervical cord CSAs of the 10 subjects at baseline were within the range of $69.13-92.12 \mathrm{~mm}^{2}$. The scan-rescan coefficient of variation (COV) was $0.617 \%$. With dehydration, we observed a mean decrease of $-0.654 \%$ (s.d. $=0.778$, $P=0.013$ ) in cord CSA as shown in Figure 2 and Table 1. After the rehydration procedure, the mean cord CSA was not significantly different from baseline (mean change $=0.121 \%$, s.d. $=1.318$ ).
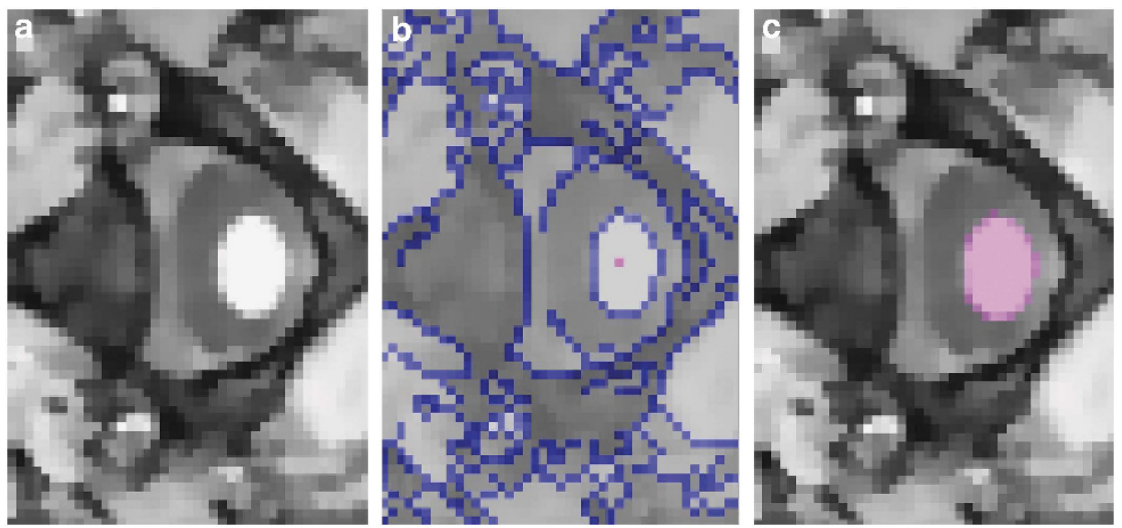

Figure 1 (a) Axial image of the cervical cord surrounded by CSF. (b) Edge map that includes a well-defined contour of the cord. The user-placed seed point is also shown. (c) Segmented cord region bounded by the edge contour. 


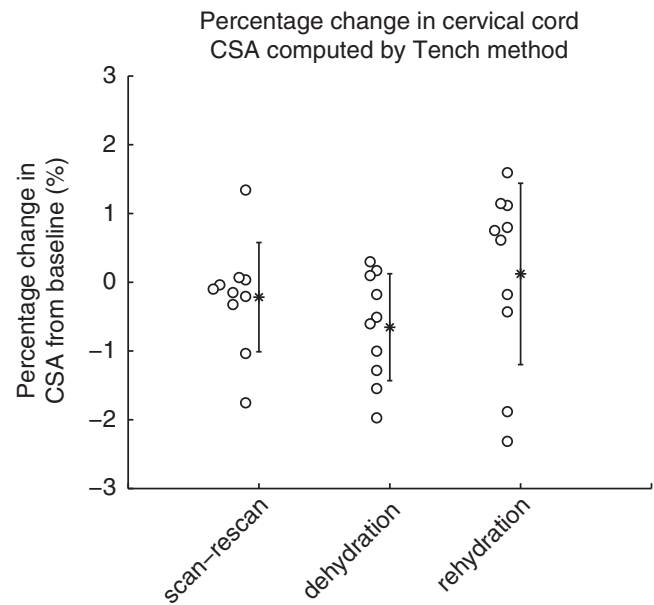

Figure 2 The percentage change in cervical cord CSA from baseline to the other three time points computed by the Tench method. The circles are individual measurements and the stars and error bar represent the means and s.d.'s. Dehydration and rehydration appear to affect CSA measurements.

Table 1 Statistical results of the percentage changes in CSA computed by the Tench and Horsfield methods

\begin{tabular}{lllll}
\hline Measurement & Method & $\begin{array}{c}\text { Mean change } \\
\%(\text { s.d. })\end{array}$ & $\begin{array}{c}\text { One-tailed } \\
\text { t-test }(\Delta>0)\end{array}$ & $\begin{array}{c}\text { One-tailed } \\
\text { t-test }(\Delta<0)\end{array}$ \\
\hline$\Delta$ Scan-rescan & Tench & $-0.217(0.794)$ & $P=0.205$ & $P=0.795$ \\
& Horsfield & $-0.452(1.197)$ & $P=0.131$ & $P=0.869$ \\
$\Delta$ Baseline to & Tench & $-0.654(0.778)$ & $P=0.013$ & $P=0.987$ \\
dehydrated & Horsfield & $-0.650(1.071)$ & $P=0.044$ & $P=0.956$ \\
$\Delta$ Baseline to & Tench & $0.121(1.318)$ & $P=0.611$ & $P=0.389$ \\
rehydrated & Horsfield & $0.057(1.129)$ & $P=0.561$ & $P=0.439$ \\
$\Delta$ Dehydrated to & Tench & $0.782(1.208)$ & $P=0.965$ & $P=\mathbf{0 . 0 3 5}$ \\
rehydrated & Horsfield & $0.715(0.939)$ & $P=0.980$ & $P=\mathbf{0 . 0 1 9}$
\end{tabular}

Abbreviation: CSA, cross-sectional area.

For both measurement methods, a reduction in cord CSA is observed after dehydration, with a return to the baseline equivalent after rehydration, as shown in the bold entities $(P<0.05)$.

However, the mean change in CSA was significant between the dehydrated and rehydrated states and was $0.782 \%$ (s.d. $=1.208$, $P=0.035$ ).

Measured using the Horsfield method, the cervical cord CSAs at baseline were within the range of $72.48-91.28 \mathrm{~mm}^{2}$, and the scan-rescan COV was $0.899 \%$. We observed a mean decrease in CSA of $-0.650 \% \quad$ (s.d. $=1.071, \quad P=0.044$ ) after dehydration compared with baseline, as shown in Figure 3 and Table 1. After rehydration, the mean CSA measurement was increased by $0.715 \%$ (s.d. $=0.939, P=0.019$ ) compared with the dehydrated state but was not significantly different from baseline (mean change $=0.057 \%$, s.d. $=1.129)$.

\section{DISCUSSION}

We investigated the effect of mild to moderate dehydration and rehydration on CSA measurements of the cervical spinal cord in healthy subjects. We have observed a decrease in cervical cord CSA after solid and liquid fasting for an overnight period that would not be considered unusual in routine research or clinical settings involving MRI.

We used two independent CSA measurement methods, each having been demonstrated to be effective in previous clinical studies, in our

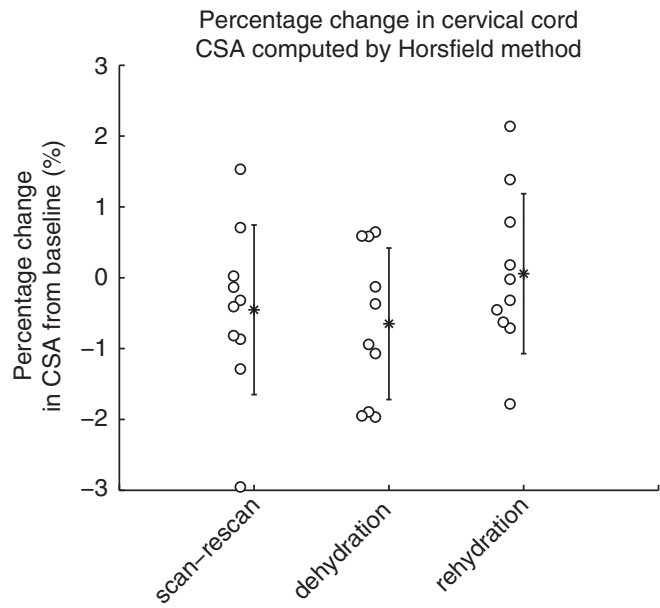

Figure 3 The percentage change in cervical cord CSA from baseline to the other three time points computed by the Horsfield method. The circles are individual measurements and the stars and error bar represent the means and s.d.'s. Dehydration and rehydration appear to affect CSA measurements.

analysis to account for any bias introduced by either method. The two methods agreed well on the mean change in cervical cord CSA observed after dehydration $(-0.654 \%$ for the Tench method and $-0.650 \%$ for the Horsfield method), which is similar to the change of $-0.55 \%$ observed by Duning et al. ${ }^{16}$ in their study on dehydration effect on whole-brain volume. In addition, the two methods agreed well on the mean change between the dehydrated and rehydrated states $(0.782 \%$ for the Tench method and $0.715 \%$ for the Horsfield method), which is similar to the $0.72 \%$ increase in whole-brain volume that Duning et al. ${ }^{16}$ found. The brain and spinal cord are directly connected and have similar mechanisms for their regulation of water balance; ${ }^{21,22}$ therefore, it is reasonable to speculate that the current results reflect the cord exhibiting an incomplete compensation to fluid deficiency, similar to what has been observed in the brain. Overall, our results lend further evidence that hydration status can affect volumetric measures of the central nervous system on MRI.

However, there are a number of limitations in our study, including the small size of the cord, the reproducibility of the measurement methods and the small sample size. To understand these limitations, it is helpful to examine the precision when using each measurement method. Horsfield et al. ${ }^{14}$ estimated the minimum area change detectable by their method using the following equation: minimum detectable change $=$ group mean $\times$ intra-scan COV $\times 1.96=0.87 \mathrm{~mm}^{2}$ for their study. Alternatively, the detectable percentage change can be estimated by multiplying the intra-scan COV by 1.96 to obtain the change that can be detected with $95 \%$ confidence interval, which yields $1.16 \%$ and $0.27 \%$ for the Horsfield and Tench methods, respectively, using values from their published studies. As the intra-scan COV is image- and operator-dependent, we also estimated the precision of the two methods with our current data, which resulted in detectable percentage changes of 1.08 and $0.09 \%$ for the Horsfield and Tench methods, respectively. Our estimated change because of dehydration was $0.65 \%$ for both methods, which meets the $95 \%$ confidence threshold of the Tench method but is below that of the Horsfield method, which favors the former method but does not preclude the possibility of the latter method detecting a systematic change. Given that the two methods agree well on both the effects of dehydration and rehydration, and that at least one is confirmed to have the necessary sensitivity on these 
data, we conclude that the change observed was likely real. It should be noted that because the Tench method was our own implementation, the operator was very familiar with the software, which may explain the better reproducibility; however otherwise, these results do not indicate that either method is superior.

Another limitation is that no brain scans (due to limited scanning time) were collected and a firm conclusion cannot be made about whether similar magnitudes of change can be expected in both structures. In addition, whereas the Duning study ${ }^{16}$ found that the mean brain volume increased beyond baseline after rehydration, the mean cord volume in our study was significantly increased only when compared with the dehydrated state, and not to baseline. A related confounding factor is the effect of brain volume changes on cord position. The cord could in theory be shifted rostrally because of the shrinkage of the brain after dehydration and because the cord does not have fixed landmarks (one could conceivably use the peripheral nerves, but they are very small and quite far apart), it would be very difficult to ensure that exactly the same level of cord is being measured. However, given that the dehydration effect on brain volume is likely to be $<1 \%$, the cord shift is likely to be correspondingly small and, in the absence of local injury, the cord's diameter varies smoothly over its length; therefore, we expect the effect of cord shift to be minor.

Whereas there is increasing evidence that hydration status is a confounding factor in the volumetric analysis of MRIs, there is little information on how to correct for such fluctuations and whether this is even possible. Traditional methods for monitoring hydration status, such as urinalysis, are unlikely to be reliable because of the complex nature of the body's mechanisms for water balance, which involves multiple systems whose health can change over time, even in normal aging. Nonetheless, studies of the hydration effect on brain and cord measures are valuable for improving the understanding of study results that may be affected by changes in water content. The results of our current study have particular implications for studies of spinal cord disorders that involve an inflammatory response. For example, previous cross-sectional studies have shown that cord volume in MS patients can be increased (with varying levels of statistical significance) when compared with healthy controls, especially in early MS. $^{3,9}$ These findings are somewhat unintuitive for a neurodegenerative disease but are hypothesized to reflect the presence of inflammation and associated edema, which can induce a temporary increase in cord volume. Our current results help to bolster that hypothesis by demonstrating that measurable volume changes are associated with fluctuations in water content. In a longitudinal study of MS patients who had a spinal cord-related relapse, ${ }^{23}$ the patients showed a decline in cervical spinal cord area of $\sim 0.7 \%$ monthly during the follow-up period of 6 months, even though they were improving clinically, which may be attributable to a resolution of inflammation and edema. Our current results show that changes of that magnitude can occur in a short period of time, and that frequent cord scanning after an acute episode can be a potentially useful method for monitoring edema. This is especially true, given that certain MS therapies, such as natalizumzab, have been shown in human subjects to have a pseudoatrophy effect on the brain ${ }^{24,25}$ and in preclinical studies to have a strong anti-inflammatory effect on the cord. ${ }^{26}$ Inflammation is also an important feature of pathology and repair for traumatic spinal cord injury ${ }^{27}$ but typically in a shorter time frame than in neurodegenerative diseases. Repeated MRIs initiated soon after the onset of trauma to measure cord volume, which may increase or decrease, may allow improved monitoring of the inflammatory and recovery processes. ${ }^{28}$ This may be especially important for evaluating the effectiveness of anti-inflammatory therapy, the timing of which can be an influential factor to the clinical outcome. ${ }^{26}$ Chronic spinal cord injury also leads to irreversible atrophy rostral to the lesion over time. ${ }^{29}$ The importance of structural MRI for understanding these changes is becoming increasingly recognized; ${ }^{28}$ however, the results of our current study are likely more applicable to the acute phase.

In conclusion, we have demonstrated that hydration status affects spinal cord CSA measurements on MRI and should be considered as a source of variability in clinical studies of spinal cord atrophy. Our results also support the use of frequent MRI scanning to monitor conditions that may involve changes in water content, such as the inflammation and edema associated with acute spinal cord injury.

\section{DATA ARCHIVING}

There were no data to deposit.

\section{CONFLICT OF INTEREST}

The authors declare no conflict of interest.

\section{ACKNOWLEDGEMENTS}

We thank the volunteers for their valuable time and effort. We thank Trudy Harris, Dr Burkhard Mädler and Dr Corree Laule for their assistance in developing the scanning protocol. This research was funded by Biogen Idec Canada and the Natural Sciences and Engineering Research Council of Canada (NSERC).

1 Zivadinov R, Bakshi R. Central nervous system atrophy and clinical status in multiple sclerosis. J Neuroimaging 2004; 14: 27S-35S.

2 Lin X, Tench CR, Eangelou N, Jaspan T, Constantinescu CS. Measurement of spinal cord atrophy in multiple sclerosis. J Neuroimaging 2004; 14: 20S-26S.

3 Klein JP, Arona A, Neema M, Healy BC, Tauhid S, Goldberg-Zimring D et al. A 3T MR imaging investigation of the topography of whole spinal cord atrophy in multiple sclerosis. Am J Neuroradiol 2011; 32: 1138-1142.

4 Evangelou N, DeLuca GC, Owens T, Esiri MM. Pathological study of spinal cord atrophy in multiple sclerosis suggests limited role of local lesions. Brain 2005; 128: 29-34.

5 Agosta F, Absinta M, Sormani MP, Ghezzi A, Bertolotto A, Montanari E et al. In vivo assessment of cervical cord damage in MS patients: a longitudinal diffusion tensor MRI study. Brain 2007; 130: 2211-2219.

6 Rovaris M, Judica E, Sastre-Garriga J, Rovira A, Sormani MP, Benedetti B et al. Large-scale, multicentre, quantitative MRI study of brain and cord damage in primary progressive multiple sclerosis. Mult Scler 2008; 14: 455-464.

7 Healy BC, Arora A, Hayden DL, Ceccarelli A, Tauhid S, Neema M et al. Approaches to normalization of spinal cord volume: application to multiple sclerosis. J Neuroimaging 2012; 22: e12-e19.

8 Bieniek M, Altmann DR, Davies GR, Ingle GT, Rashid W, Sastre-Garriga J et al. Cord atrophy separates early primary progressive and relapsing remitting multiple sclerosis. J Neurol Neurosurg Psychiatry 2006; 77: 1036-1039.

9 Mann RS, Constantinescu CS, Tench CR. Upper cervical spinal cord cross-sectional area in relapsing remitting multiple sclerosis: Application of a new technique for measuring cross-sectional area on magnetic resonance images. J Magn Reson Imaging 2007; 26: 61-65.

10 Rashid W, Davies GR, Chard DT, Griffin CM, Altmann DR, Gordon R et al. Increasing cord atrophy in early relapsing-remitting multiple sclerosis: a 3 year study. J Neurol Neurosurg Psychiatry 2006; 77: 51-55.

11 Furby J, Hayton T, Altmann D, Brenner R, Chataway J, Smith KJ et al. A longitudinal study of MRI-detected atrophies in secondary progressive multiple sclerosis. J Neurol 2010; 257: 1508-1516.

12 Losseff NA, Webb SL, O'Riordan JI, Page R, Wang L, Barker GJ et al. Spinal cord atrophy and disability in multiple sclerosis. A new reproducible and sensitive MRI method with potential to monitor disease progression. Brain 1996; 119. 701-708.

13 Tench CR, Morgan PS, Constantinescu CS. Measurement of cervical spinal cord cross-sectional area by MRI using edge detection and partial volume correction. J Magn Reson Imaging 2005; 21: 197-203.

14 Horsfield MA, Sala S, Neema M, Absinta M, Bakshi A, Sormani MP et al. Rapid semi-automatic segmentation of the spinal cord from magnetic resonance images: application in multiple sclerosis. Neurolmage 2010; 50: 446-455.

15 Sampat MP, Healy BC, Meier DS, Dell'Oglio E, Liguori M, Guttmann CR. Disease modeling in multiple sclerosis: assessment and quantification of sources of variability in brain parenchymal fraction measurements. Neuroimage 2010; 52: 1367-1373. 
16 Duning T, Kloska S, Steinstrater O, Kugel H, Heindel W, Knecht S Dehydration confounds the assessment of brain atrophy. Neurology 2005; 64 548-550.

17 Dickson JM, Weavers HM, Mitchell N, Winter EM, Wilkinson ID, Van Beek EJR et al. The effects of dehydration on brain volume -preliminary results. Int J Sports Med 2005; 26: 481-485.

18 Kempton MJ, Ettinger U, Schmechtig A, Winter EM, Smith L, McMorris T et al. Effects of acute dehydration on brain morphology in healthy humans. Hum Brain Mapp 2009; 30: 291-298.

19 Kempton MJ, Ettinger U, Foster R, Williams SCR, Calvert GA, Hampshire A et al. Dehydration affects brain structure and function in healthy adolescents. Hum Brain Mapp 2011; 32: 71-79.

20 Streitbürger DP, Möller HE, Tittgemeyer M, Hund-Georgiadis M, Schroeter ML, Mueller K. Investigating structural brain changes of dehydration using voxel-based morphometry. PLOS ONE 2012; 7: e44195.

21 Saadoun S, Papadopoulos MC. Aquaporin-4 in brain and spinal cord oedema. Neuroscience 2010; 168: 1036-1046.

22 Benga O, Huber VJ. Brain water channel proteins in health and disease. Mol Aspects Med 2012; 33: 562-578.
23 Ciccarelli O, Altmann DR, McLean MA, Wheeler-Kingshott CA, Wimpey K, Miller DH et al. Spinal cord repair in MS: does mitochondrial metabolism play a role? Neurology 2010; 74: 721-727.

24 Zivadinov R, Reder AT, Filippi M, Minagar A, Stüve O, Lassmann $\mathrm{H}$ et al. Mechanisms of action of disease-modifying agents and brain volume changes in multiple sclerosis. Neurology 2008; 71: 136-144.

25 Miller DH, Soon D, Fernando KT, MacManus DG, Barker GJ, Yousry TA et al. MRI outcomes in a placebo-controlled trial of natalizumab in relapsing MS. Neurology 2007; 68: 1390-1401.

26 Fleming JC, Bao F, Chen Y, Hamilton EF, Gonzalez-Lara LE, Foster PJ et al. Timing and duration of anti-alpha4betal integrin treatment after spinal cord injury: effect on therapeutic efficacy. J Neurosurg Spine 2009; 11: 575-587.

27 Fleming JC, Norenberg MD, Ramsay DA, Dekaban GA, Marcillo AE, Saenz AD et al. The cellular inflammatory response in human spinal cords after injury. Brain 2006; 129: 3249-3269.

28 Freund $\mathrm{P}$, Curt A, Friston K, Thompson A. Tracking changes following spinal cord injury: insights from neuroimaging. Neuroscientist 2013; 19: 116-128.

29 Freund P, Weiskopf N, Ward NS, Hutton C, Gall A, Ciccarelli 0 et al. Disability, atrophy and cortical reorganization following spinal cord injury. Brain 2011; 134: 1610-1622. 